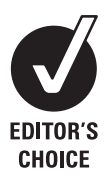

${ }^{1}$ Departments of Pathology and Laboratory Medicine, and Internal Medicine, Walther Oncology Center, Indiana University School of Medicine, Indianapolis, Indiana, USA; ${ }^{2}$ Departments of Surgery, Biochemistry and Molecular Biology, Walther Oncology Center, Indiana University School of Medicine, Indianapolis, Indiana, USA

Correspondence to: Sunil Badve, Clarian Pathology Laboratory, 350 West 11th

Street, 4th Floor, Indianapolis, IN 46202, USA; sbadve@iupui.edu

Accepted 14 August 2008 Published Online First 15 September 2008

\title{
Oestrogen-receptor-positive breast cancer: towards bridging histopathological and molecular classifications
}

\author{
S Badve, ${ }^{1}$ H Nakshatri
}

\section{ABSTRACT}

The oestrogen receptor (ER) pathway is key for survival and progression in a significant proportion of breast cancers. The ER can be activated by oestrogen or activated due to "crosstalk" with growth factor receptor pathways. Activated ER signals through transcriptional and non-transcriptional mechanisms.

Immunohistochemistry (IHC), in spite of the shortcomings, remains the method of choice as it provides for in situ assessment of ER expression within the tumour cells. This capability is lost in tissue grinding methods that assess oestrogen-binding activity or messenger RNAs in tumours. $\mathrm{IHC}$ is also not influenced by the presence of non-tumoural cells or low amounts of tumour cells within samples examined. It is clear that ER-positive tumours do not represent a single entity. Irrespective of the terminology used, low-grade ER-positive (also known as luminal A) tumours need to be differentiated from high-grade/highly proliferative ER-positive tumours. This can be done in a variety of ways including but not limited to analysis of FOXA1 and GATA-3 by IHC, and limited molecular profiling by Oncotype DX, MGH2-gene signature, intrinsic gene signature or MapQuant Dx. Several areas of ER biology are still poorly understood; these include: its function in the cytoplasm/plasma membrane, its role in the differentiation to proliferation switch, and pathways associated with resistance to hormonal therapy. A detailed understanding of these areas will permit better classification and a personalised approach to management of ER-positive breast cancers.

Steroid hormones, in particular oestrogens and progesterones, are crucial for normal growth and development of the mammary gland, and also as growth factors for the large majority of breast cancers. ${ }^{12}$ Expression of oestrogen receptors (ERs) in breast cancer is a prognostic marker, and also a predictive marker of response to therapies that target oestrogen synthesis (oophorectomy or aromatase inhibitors) or those that block the action of its receptor (eg, tamoxifen). However, resistance to endocrine therapy, innate or acquired, is common. Studies in the last decade have begun to help in the understanding the biological pathways associated with resistance. This review will present state of art knowledge about the biology of ERs with particular reference to tumour classification and assessment in clinical practice.

\section{Biology of ERs}

ER gene and protein

The action of oestrogen is mediated by its receptors (ERs), which dimerise after binding to oestrogen and bind the DNA at sites known as ER-responsive elements (EREs) or by piggybacking on other transcription factors (detailed later). DNA-bound ER then recruits a series of protein complexes, and this leads to either increased or decreased gene transcription. There are two forms of ER, $\alpha$ and $\beta$, which together mediate downstream events. ER $\alpha$ (classical ER), the major player in breast cancer, is analysed in clinical practice. Several isoforms of ER $\alpha$ exhibiting variable functions exist; however, their role in breast cancer, particularly in antioestrogen resistance, remains controversial. ER $\beta$, on the other hand, is a relatively newly recognised $\mathrm{ER}^{3-5}$ and its function, particularly its role in breast cancer, is poorly understood. This has been largely because of the poor quality of reagents and the presence of at least five isoforms. ${ }^{6}$ ER $\beta$ has not entered into the routine clinical practice of breast cancer (and it is mostly excluded from the current review). However, it must be noted that ER $\beta$ variants have been associated with prostate cancer risk. ${ }^{7}$

The ER $\alpha$ gene comprises six functional domains encoded by eight exons and it encodes a major $67 \mathrm{kDa}$ protein; small variants generated through alternative splicing or promoter usage have been described. The centrally located DNA-binding domain and nuclear localisation signal are flanked on either side by transactivation domains AF-1 and AF-2 (fig 1). The activity of the AF-1 domain is of great interest for breast cancer because it can be activated by phosphorylation by a number of growth-factor-induced kinases, including those commonly activated in breast cancer such as MAP kinase and AKT/PKB. ${ }^{9}$ These phosphorylation events generally lead to non-oestrogen-dependent ER activity or enhancement of oestrogendependent activity. The importance of recognising these two separate activation function domains is that tamoxifen predominantly acts by inhibiting the function of the AF-2 domain. ${ }^{10}$ Tamoxifen is a selective oestrogen modulator that functions as an antagonist (as in breast) or partial agonist (in bone and endometrium) of the ER depending on the cell type. The growth-factor-activated signals that modulate the function of AF-1 can dominantly impair the ability of tamoxifen to block AF-2 function in breast and thus reduce tamoxifen response and/or convert tamoxifen to an agonist (see below). In contrast, fulvestrant, a pure antioestrogen, blocks ER function by causing receptor degradation. ${ }^{11}$

In the absence of oestrogen, ER is held in a complex of co-repressor proteins, which are 


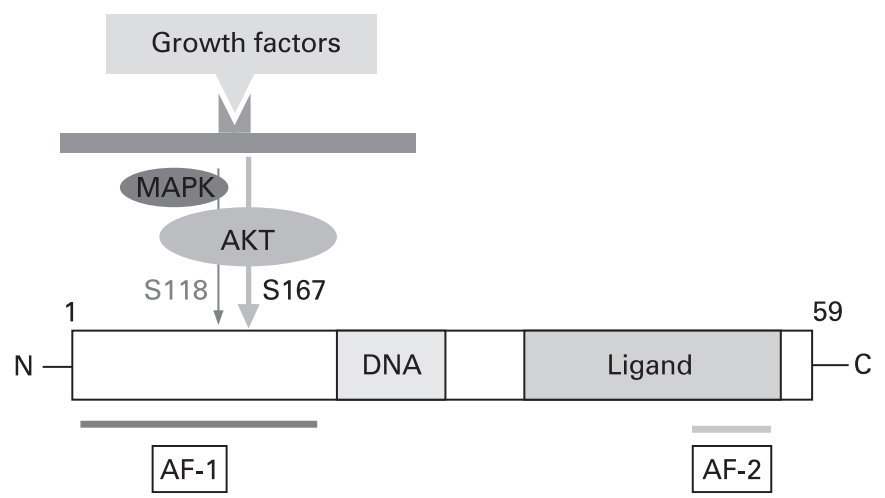

Figure 1 Structure of the oestrogen receptor $(E R) \alpha$ gene is shown with its DNA-binding domain and activating function (AF)-1 and AF-2 domains. Also note that the ER can be activated even the absence of oestrogen by growth factor pathways by phosphorylation of serine residues S118 and S167 by MAPK and AKT, respectively.

replaced by co-activator proteins upon binding with oestrogen. At least 100 proteins are known to interact with $\mathrm{ER} \alpha$; among these are at least a dozen proteins are described as co-activators or co-repressors. ${ }^{12}$ The NCOA family (NCOA1-NCOA3) and the NCOR family (NCOR1 and NOCR2/SMRT) represent major co-activators and co-repressors, respectively. ${ }^{13}{ }^{14}$ Genetic deletion experiments in animal models have clearly shown the relevance of these co-activator/co-repressor molecules in oestrogen signalling. Thus, receptor levels as well as balance between co-activators and co-repressors determine overall receptor activity in cancer cells. Given below are details of a few relevant studies that have addressed the role of ER co-regulators in breast cancers, although there is limited consensus on which of these molecules can be brought to routine clinical practice. (i) Amplification as well as overexpression of amplified in breast cancer 1 (AIB1, also called NCOA3), a co-activator protein thought to play a critical role in integrating growth factor pathways with ER:oestrogen signalling pathways, has been described. ${ }^{15}{ }^{16}$ In a study by Osborne et al, AIB1 overexpression along with HER2 overexpression in ER-positive breast cancer was associated with tamoxifen resistance and decreased survival. However, unlike this previous study, we did not find expression of AIB1 to correlate with outcomes. ${ }^{16}$ (ii) Ellis and coworkers have analysed the expression of several co-regulators (NCOA1/SRC1, NCOA3/AIB1, NCOR2/SMRT and CBP/ p300) in a series of breast cancers. They found that although NCOA1/SRC1 expression was associated with longer overall survival and disease-free interval, only NCOR2/SMRT expression was an independent prognostic factor predicting poor outcome. $^{17}$

\section{ER functional pathways}

The first decade of ER research, subsequent to cloning in 1985, focused primarily on structure-function studies. Identification of co-regulator molecules that control ER activities, and subsequent studies involving epigenetic regulation of gene expression and ER:growth factor signalling crosstalk, enabled broad classification of ER actions to genomic and non-genomic action. The genomic action can be further classified into classical and non-classical. These three functions of ER work in concert to contribute to the overall effect of oestrogen on proliferation of ER-positive breast cancers and more importantly resistance to antihormonal therapy.

\section{Genomic pathways}

In the classical genomic pathway, following ligand binding, the homodimerised receptor complex binds to specific EREs located in the regulatory regions of target genes, controlling their level of transcription. This pathway is modulated by crosstalk/ interaction with transcription factors activated by other signal transduction pathways, heterodimerisation with ER $\beta$ and ER $\alpha$ isoforms, and binding of ER $\alpha$ to non-DNA-binding co-activator and co-repressor proteins.

In addition to the classical pathway, ER can affect transcription of a number of genes that do not contain EREs. Only $40 \%$ of oestrogen-regulated genes in breast cancer cell lines contain ERE-binding sites (H Nakshatri, unpublished data). Oestrogenregulated expression of non-ERE-containing genes is achieved via interaction with other transcription factors such as specificity protein 1 (Sp-1) and members of the Fos/Junactivating protein 1 (AP-1) pathway. ${ }^{18}{ }^{19}$ The current estimate is that $\sim 70 \%$ of oestrogen-regulated genes are repressed after oestrogen treatment; in most cases this is believed to be mediated via interaction of ER with other transcription factors. ${ }^{20}$

\section{Non-genomic rapid ER activity}

For a detailed discussion of these pathways see Levin and Pietras. ${ }^{21}$ Briefly, as a result of as yet poorly understood mechanisms, ER (identical to nuclear ER) is detected in the cytoplasm and cell membrane particularly in association with lipid rafts or caveolae ${ }^{22}$ where growth factor receptors such as IGFR1, EGFR and HER2 and G-coupled receptors reside. It is believed that oestrogen promotes interactions between these cell surface signalling molecules and $\mathrm{ER}$, and this leads to rapid $(<5 \mathrm{~min})$ activation of ERK and PI3 kinase. ${ }^{23}$ Activated ERK and AKT then phosphorylate nuclear ER, and upregulate its transcriptional activity and stability. This growth factor and ER pathway interaction (referred to as crosstalk) is suggested to be involved in resistance to tamoxifen. ${ }^{24}$ In vitro studies have shown that breast cancer cell lines resistant to tamoxifen upregulate HER2 and EGFR expression. ${ }^{25}$ Consistent with this, overexpression of HER2 in ER-positive patients is associated with resistance to tamoxifen and aromatase inhibitors. ${ }^{26}{ }^{27}$

There are additional reports linking crosstalk between growth factors and ER to tamoxifen resistance. Activation of protein kinase A or p21-activated kinase 1 by G-coupled receptors can lead to phosphorylation of ER at serine 305; this has the potential to convert tamoxifen from an antoagonist to an agonist. This pathway can also lead to phosphorylation of coactivators, which further increase ER activity. These effects of growth factors on ER, coupled with their additional direct effects on pathways such as the MAPK pathway and induction of AP-1 regulated genes, including Cox 2 and protein kinase $\mathrm{C} \alpha$, potentially contribute to tamoxifen resistance. We have previously shown that expression of PKC $\alpha$ in breast cancer is associated with development of resistance to tamoxifen. ${ }^{28}$

\section{Downstream events of ER activation}

Recent studies have taken advantage of the stabilising effect of formalin on DNA-protein binding to understand interactions and mechanisms of action of intranuclear proteins including transcription factors (chromatin immunoprecipitation). More recently, these assays have been further modified to extract the protein-bound DNA and identify the DNA fragments using microarrays. These "ChIP on Chip" (chromatin immunoprecipitation on microarray chips) studies have identified a large 
number of oestrogen-responsive elements $(\sim 5000)$ within the genome. ${ }^{29}$ These include genes that do not respond to oestrogen at the level of transcription; the question then arises as to what mechanisms are responsible for binding of ER to any given location (ERE) within the genome. These genome-wide studies led to the concept of a "cell lineage specific hormonal network" that controls binding specificity of ER to the genome. The transcription factors GATA-3 and FOXA1 have emerged as key components of this hormonal network. ${ }^{29}$ Most importantly, as detailed below, a subgroup of primary breast cancers with this intact hormonal network constitutes a distinct prognostic subgroup.

\section{GATA-3}

Members of the GATA family of transcription factors are expressed in a development-stage specific manner. ${ }^{30}$ The highly conserved zinc finger motif is suggested to possess a chromatindisrupting function. Although the GATA proteins are not as effective as FOXA1 in binding to nucleosomal array and open chromatin, they may cooperate with FOXA1 in efficient chromatin opening and in making regulatory regions accessible during the initial step of transcription. ${ }^{31}$ The functional cooperativity between GATA-3, FOXA1 and ER may be necessary for normal mammary gland development, particularly differentiation of mammary stem/progenitor cells to ERpositive lineage (fig 2). Deregulation of this cooperative activity may be one of the early events in ER positive breast cancers. Consistent with this possibility, GATA-3 has emerged as a strong predictor of tumour grade, ER status, differentiation status, metastasis potential and prognosis in breast cancer. ${ }^{32-35}$

\section{F0XA1}

FOXA1 (or HNF3 $\alpha$ ), a member of the "winged helix" family of transcription factors, was originally characterised for its role in liver development. ${ }^{36}$ FOXA1 can bind to chromatinised DNA and open the chromatin for binding of additional transcription factors, ${ }^{31}$ and hence it has been dubbed as a "pioneer factor." By binding to specific regions of the chromatin, it creates an "epigenetic signature" that enables transcription factors such as ER to establish a transcription programme. ${ }^{37}$ Consistent with this possibility, FOXA1-binding sites are detected on $50 \%$ of genes that are regulated by ER, and depletion of FOXA1

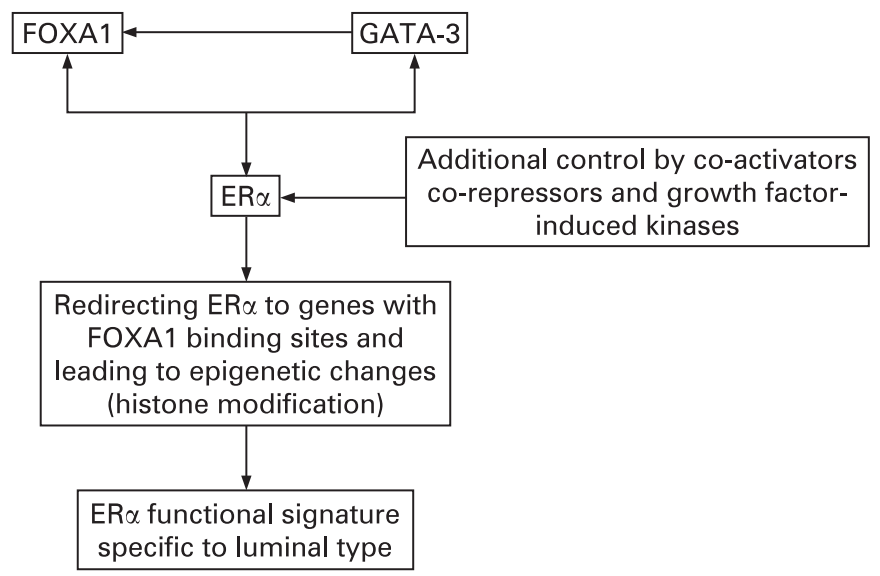

Figure 2 Inter-relationship between key members of the oestrogen receptor (ER) $\alpha$ pathway leading to functional $E R \alpha$ signature. Presence of an intact ER/GATA-3/FOXA1 network appears to be critical for the hormone responsive phenotype. partially attenuates the oestrogen response in breast cancer cells. $^{29}{ }^{38}$ Thus, one can envision ER-positive breast cancers, depending on the presence or absence of FOXA1, expressing distinct sets of oestrogen-responsive genes despite having similar levels of ER $\alpha$. FOXA1, GATA-3 and ER cooperativity observed in cell culture experiments as well as in mammaryspecific knockout studies led us and others to examine the expression pattern of these three transcription factors in primary breast cancers and relate their expression to prognosis; these studies are described below.

\section{F0XA1 and breast cancer}

Our group, ${ }^{39}{ }^{40}$ among others, ${ }^{41}{ }^{42}$ has examined the importance of FOXA1 in breast cancer using tissue microarrays, in collaboration with investigators at the University of British Columbia, Vancouver, Canada, ${ }^{39}$ and the Royal Marsden Hospital, London. ${ }^{40}$ The data showed that FOXA1 high (score greater than 3 by immunohistochemistry (IHC)) was associated with ER positivity, GATA-3 positivity and most significantly improved event-free survival compared with patients in the FOXA $1_{\text {low }}$ group; this difference was significant even at 20 years in the British Columbia study. When the analysis for stratification was based on lymph node positivity, FOXA1positive patients in node-positive and unknown nodal status groups had a better prognosis than patients who were FOXA1 negative. FOXA1 expression was found to be a better predictor of survival than progesterone receptor (PR). These data were more recently confirmed by the Ellis group in a series of $\sim 850$ patients. ${ }^{42}$ It is important to note that a specific subpopulation of normal luminal epithelial cells express FOXA1 in levels similar to those in the FOXA1 $1_{\text {high }}$ group of patients. Therefore, FOXA1 expression in breast cancers may indicate cell type origin and differentiation status of breast cancer rather than cancer-specific overexpression. A schematic representation of the hormonal network comprising ER, FOXA1 and GATA-3 in breast cancer is presented in fig 3 .

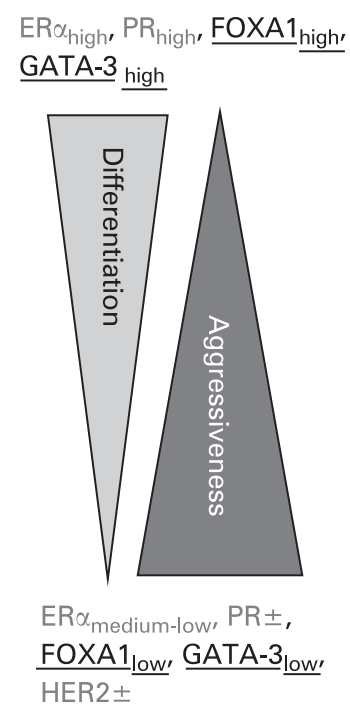

Markers in red are in current clinical use

Markers underlined may be incorporated in future

Figure 3 The role of oestrogen receptor (ER) $\alpha$ and related downstream proteins in modulating the behaviour of ER-positive breast tumours. 


\section{What determines the prognostic value of ERo}

ER-positive cells, in the normal breast, do not show proliferative activity. In contrast, ER-positive cells in atypical ductal hyperplasia, ${ }^{43} 44$ and in invasive and in situ carcinoma, show proliferative activity. The change from an ER-positive nonproliferating cell phenotype to an ER-positive proliferating cell phenotype appears to be a critical switch and is one of the characteristic events of malignancy. The exact mechanism that is responsible for this switch is as yet not understood, although deregulation of transforming growth factor $\beta$ signalling is believed to be involved. ${ }^{45}$

Additionally, not all ER-positive breast cancers are created equal. Ever since ER emerged as a major prognostic marker, there were efforts to use additional markers that could evaluate functionality of ER in tumours. One such marker is PR because its expression is directly regulated by oestrogen. A brief description of strengths and weaknesses of current assays to evaluate ER and PR is given below, and this is followed by our thoughts on new IHC-compatible markers that define ER functionality.

\section{Progesterone receptor}

$\mathrm{PR}$ has two isoforms, $\mathrm{A}$ and $\mathrm{B}$; the ratio between these two isoforms has prognostic implications. ${ }^{46}$ However, routine assays provide quantitative estimation of total amount of PR but not specific levels of two isoforms. Historically, PR analysis has been carried out in order to assess the integrity of the ER pathway; tumours that have an intact pathway (ER positive and PR positive) have a better prognosis and are more likely to be responsive to antihormonal therapy. However, our recent analysis indicate that FOXA1 is better than PR in predicting prognosis, suggesting that routine clinical analysis of ER and PR may not be sufficient. ${ }^{39}$

Most PR-positive tumours coexpress ER; however, approximately $5 \%$ of tumours are ER negative. This can be at least partially attributed to shortcomings of IHC methods since the finding is rare $(\sim 0.2 \%)$ when using reverse transcriptase (RT)PCR methods. ${ }^{47}$ In these cases, PR expression may be driven, not by ER, but by crosstalk with growth factor receptor pathways. The biology of ER-negative/PR-positive tumours is poorly understood; depending upon the study, these tumours can be responsive or resistant to endocrine therapy.

Loss of PR expression is not uncommon in ER-positive /PRpositive tumours that have metastasised to distant sites. This loss is often associated with development of resistance to endocrine therapy. ${ }^{48}$ Similar loss of ER at metastatic sites has been reported $^{49}$ and patients who have discordant receptor status between primary and metastatic sites have an aggressive clinical course as compared with patients whose tumours are concordant. ${ }^{50}$ It is not yet clear if detailed analysis of downstream targets of ER will allow early identification of ERpositive /PR-positive primary tumours with a non-functional ER/PR pathway and thus proclivity for metastasis.

\section{Assays for ER and PR}

In the past, ligand binding assays that measured radiolabelled oestrogen-binding activity were performed on ground-up frozen tumours. Apart from the use of radioactivity, significant handicaps included the requirement of fresh frozen tumours and the lack of morphological data within these tissue samples. The grossly selected tissue samples were invariably a mixture of tumour cells and benign (ER-positive) epithelium, resulting in false-positive results. Similarly, false-negative results were seen when the tissue contained very few ER-positive cells. This is borne out by the fact that in many of the studies conducted during this period, a small fraction of ER-negative tumours showed response to antihormonal therapy. The availability of antibodies that can detect the ER epitope in archival formalinfixed paraffin-embedded (FFPE) tissues has enabled the use of IHC for ER and PR analysis. Although IHC has been used for a long period of time, it is still plagued by a number of technical issues leading to discordant results.

\section{Newer ER assays}

As stated above, IHC is still the most widely used method for detection/quantitation of ER. The amount of ER detected depends upon preanalytical and postanalytical variables including fixation times, protocol for processing, and antibodies used. IHC using 1D5 or 6F11 mouse monoclonal antibodies has been the most widely used assay to assess the efficacy of antihormonal therapy. Cheang et al, ${ }^{51}$ in a study involving over 4000 patients, demonstrated the utility of SP1 rabbit monoclonal antibody, particularly in relation to prognosis. In that study, SP1 detected approximately $8 \%$ more cases as ER positive. ${ }^{51}$ It is currently controversial whether SP1 antibody is a better reagent. It is possible that this reagent is a little too sensitive, as it picks up ER-positive cells in tumours that are not conventionally regarded as ER positive. An example is lung cancer where it is not unusual for SP1 to be positive in a small percentage of cells. Despite this limitation, many commercial kits including PharmDX kit contain rabbit ER antibodies. Currently there are a lack of data validating the true significance of the increased staining seen with rabbit monoclonal antibodies.

In quantitative RT-PCR, RNA is extracted from thick sections of FFPE blocks, and ER mRNA levels are analysed. The "linear" data obtained can be normalised; this is considered significant improvement over IHC methods. However, these methods are similar to ligand-binding assays with regard to the need to grind tissue. The data obtained, like IHC, also depend on preanalytical variables such as fixation, methods used for extraction, and the choice of probes used. These issues have not been well analysed and need additional studies.

We and others have shown that under standard assay conditions, there is an extremely high degree of correlation between RT-PCR analysis and IHC (performed at a local institution or at a central coordinating pathology laboratory using clone 1D5) for ER and clone 636 for PR. ${ }^{52}{ }^{53}$ These data would suggest that inaccuracies of IHC assay are grossly exaggerated.

\section{What is ER positive?}

This almost idiotic question is unfortunately difficult to answer. The easier question to answer is what is ER negative? Tumours that do not have/show any expression on IHC are clearly negative. It is controversial as to what level of expression determines the true cut-off point in terms of response to antihormonal therapy. In 2000, the National Institute of Health Cancer panel recommended that tumours that contain any ERpositive nuclei should be considered as ER positive. This was partly based on the studies by Harvey et al in a large series of cases in which 6F11 antibody was used to analyse ER expression..$^{55}$ This study showed that the level of ER expression as measured by intensity and percentage of staining (Allred Score) strongly correlated with outcome. However, the tissue samples used were remnants from old ligand-binding studies and were present in the form of ground pellets. ${ }^{55}$ 
Additionally, a control arm was lacking. In spite of these limitations, the study was a major advance in that it established the need for analysis of intensity and percentage of positive cells; this is now a part of most ER/PR IHC analyses.

More recently, quantitative RT-PCR (qRT-PCR) analysis has been retrospectively performed on old National Surgical Adjuvant Breast and Bowel Project (NSABP) clinical trial samples (B-20 and B-14); the same studies from which the Oncotype DX recurrence score was developed. ${ }^{56}{ }^{57}$ Expression of ER was classified based on RNA analysis into tertiles. These data clearly showed marked benefit of antihormonal therapy in patients with the highest levels of ER; however, there was little or no benefit in patients within the lowest tertile. This seems to suggest that classifying a tumour as ER positive when it contains very few positive nuclei may be incorrect. Additional studies are required to further clarify these issues. Incidentally, these RT-PCR studies confirmed the prognostic utility of PR analysis in ER-positive patients.

\section{Cut-off point for clinical report}

Although one can argue about the validity of cut-off points used for the purposes of a clinical report, it is important to have a uniform method for data collection. In our laboratory, we report intensity and percentage of staining; this is in keeping with a recent ad hoc IHC standardisation committee recommendation (unpublished). Cases that do not show any nuclear ER positivity $(0 \%)$ are classified as negative. In our experience with in-house clinical cases, we seldom see marked variation in intensity of staining; however, this is not true when analysis is done on outside cases or tissue microarrays from clinical trial materials. This might indicate that preanalytical variables affect intensity of staining.

\section{Newer issues related to ERs and PRs}

Bridging histopathological and molecular classifications

Perou et al, using cDNA microarrays, classified breast cancer into five subtypes, namely luminal A and B, HER2, and basal and normal subtypes. ${ }^{58}$ Non-luminal A/ER-positive tumours have been subclassified into luminal $B$ and luminal $C$ phenotypes. It should be noted that none of these categories was defined using IHC methods. In most studies, luminal A phenotype is defined to have the following characteristics: (i) expressing ER and PR, (ii) strongly associated with low histological grade and proliferative activity, (iii) coexpressing FOXA1 and GATA3, and (iv) good prognosis with $>95 \%$-year survival. Luminal B tumours are defined as tumours that coexpress to ER and HER2. More recently, Cheang et al have defined luminal $\mathrm{B}$ tumours as ER positive with high proliferative activity (KI 67 greater than 19\%) and another category of tumours that are ER positive and HER2 positive. ${ }^{59}$ In their recent analysis of over 4000 cases, ER-positive tumours (by IHC) with high proliferation had the worst outcome. ${ }^{59}$ It is likely that these tumours do not have a functional ER. In addition, at least some of these tumours show an overlap with basal tumours in gene expression profiling studies ( $\mathrm{C}$ Perou, personal communication). A simple algorithm showing the relationship between molecular classification and traditional histological classification is shown in fig 4.

Although molecular classification is a significant advancement, it has a number of limitations. High costs and patent issues have significantly limited its use. The intrinsic gene signature is being commercially developed. ${ }^{60}$ This will enable widespread use and independent assessment of the value of the intrinsic classification. It is possible that the analysis of a large number of cases might lead to further modifications of the classification. Another important point to remember is that lobular carcinoma (classical, ILC) as per molecular classification falls in the same category as low-grade invasive ductal carcinoma (IDC) and is not recognised as a separate entity. The biological behaviour of ILC including pattern of metastasis is clearly distinct from low-grade IDC; this clearly merits its recognition as a separate entity.

\section{Molecular tests for predictive assessment of ER-positive tumours} cDNA microarrays have generated signatures for ER-positive breast cancers contain more than 10 genes, and none have been reduced to simple protein expression analysis of a few markers. The Oncotype DX recurrence score (RS) is one of the measures that can be used to assess sensitivity to hormonal therapy. ${ }^{56}{ }^{57}$ This RT-PCR assay examines the expression of 16 genes (plus five control genes) in FFPE tissues. Among the genes in this assay, levels of ER, HER2 and proliferation-related genes significantly influence the overall score, although other genes also play a role. As one would predict, most patients who are HER2 positive end up in the intermediate or high-risk categories. The RS of this assay was originally developed in NSABP B-14 and B-20 clinical trials, which included only nodenegative patients. Additional recent studies using materials from E2197 and S8814 randomised clinical trials showed that this assay can effectively predict prognosis even in node-positive patients. ${ }^{53}{ }^{61}$ Patients with a low RS are more likely to respond to hormonal therapy alone, and those with a high RS derive significant benefit from chemotherapy. Currently in the USA, patients with an intermediate RS are being enrolled in a randomised clinical trial (TAILORx) to evaluate the need for chemotherapy. Although there is a rush to use the Oncotype DX test in clinical practice, most of the studies to date have failed to show a significant superiority of this test over Scarff Bloom Richardson histological grade. ${ }^{56}$ However, the test offers a significant advantage over histological grade in terms of precision, and offers a continuous parameter for risk assessment.

Multiple other assays are being developed specifically for prognostication of ER-positive tumours; these include the MGH2-gene signature. This commercially available assay analyses risk based on the ratio of two genes (Hox13 and IL17BR) ${ }^{62-64}$ More recently, another molecular test, MapQuant Dx (previously called genomic grade index), which combines the expression levels of genes that discriminate between histological grade 1 and grade 3 tumours, has become commercially available. ${ }^{656}$ Interestingly, several of the genes in this test are downstream of ER signalling and may additionally evaluate ER functionality in tumours (S Badve, unpublished observations).

\section{Novel predictive markers using IHC assays}

The ER pathway is extremely complex and contains: (i) coactivator and co-repressor proteins, (ii) transcription factors that modify binding of ER to its targets, and (iii) crosstalk activation due to links to the growth factor receptor pathways. This is particularly evident in cases with HER2 overexpression; ER-positive tumours that coexpress HER2 are more likely to exhibit resistance to endocrine therapy. ${ }^{26}{ }^{27}$ Therefore, it is important to develop additional markers suitable for IHC that can define ER functionality in face of multiple cancer-specific alterations including HER2 amplification in ER-positive tumours. Based on the basic research and subsequent correlation studies by several groups including ours, we propose a model 
Figure 4 Rough guide to molecular classification of breast cancer in relation to grade and oestrogen receptor (ER) status.

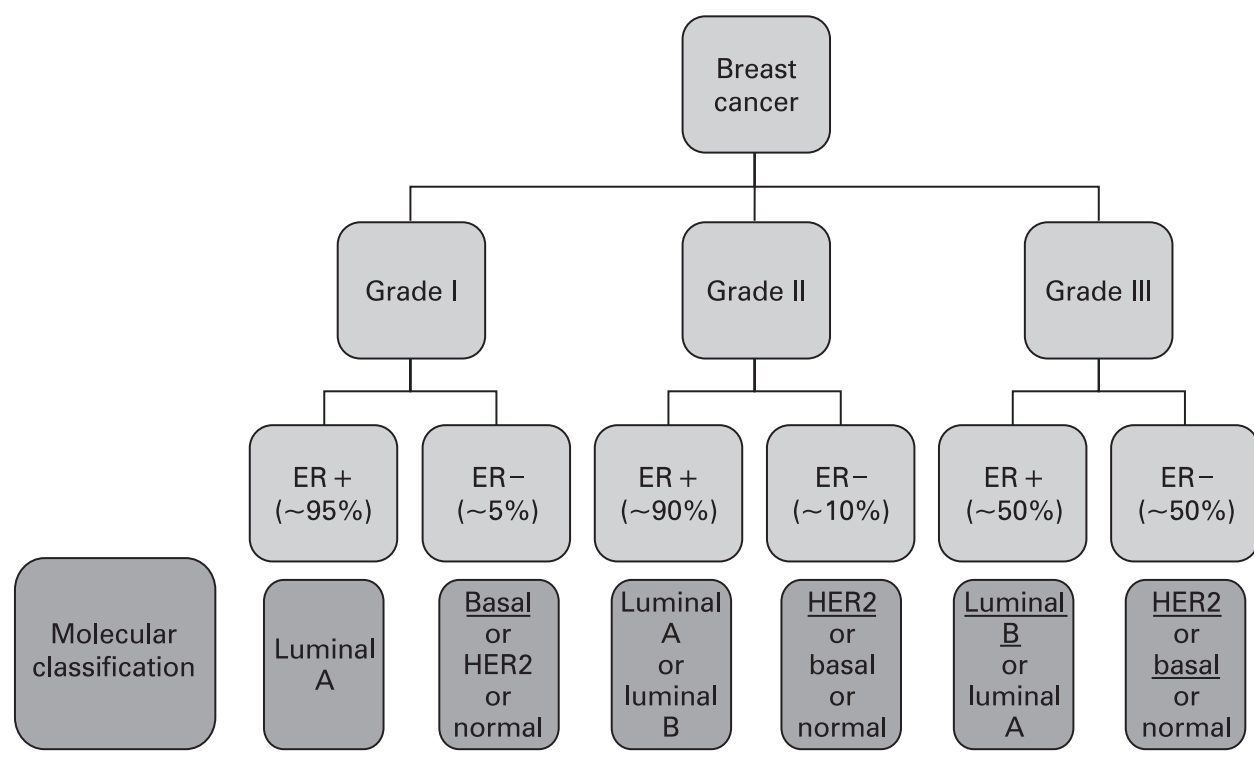

Predominant type is underlined. that the hormonal network comprising ER, FOXA1 and GATA3 constitutes a predictive and prognostic signature for ERpositive breast cancers (fig 2). GATA-3 is essential for the expression of FOXA1, and FOXA1 is involved in redirecting ER to specific regions of the chromosome. ${ }^{37}{ }^{67}$ GATA-3 is required for the expression of genes linked to luminal differentiation such as whey acidic proteins, $\alpha$-lactalbumin and several caseins. ${ }^{68}$ Thus, cancer cells expressing ER but lacking GATA-3 may not express all differentiation-associated genes. Similarly, cancer cells expressing ER but lacking FOXA1 may express very few of the oestrogen-responsive genes and have evolved to grow independent of oestrogen.

The expression of other markers in the ER pathway, particularly co-regulators, has provided some additional information but it is unlikely that these might have any role to play in clinical practice in the near future (figs 3 and 4).

\section{SUMMARY AND CONCLUSIONS}

The ER pathway is key for survival and progression in a significant proportion of breast cancers. ER activation can be due to oestrogen or due to crosstalk with growth factor receptor pathways and it signals through transcriptional and nontranscriptional mechanisms. IHC, in spite of the shortcomings, remains the method of choice for assay of ER, as it provides direct assessment of expression within the tumour cells. It is not dependent upon extrinsic factors such as non-tumoural epithelial cells or low numbers of tumour cells within samples examined.

It is clear that ER-positive tumours do not represent a single entity. Irrespective of terminology used, low-grade ER-positive (also known as luminal A) tumours need to be differentiated from high-grade/highly proliferative ER-positive tumours. This can be done by a variety of ways including but not limited to analysis of FOXA1, GATA-3, Oncotype DX, intrinsic gene signature, MGH2-gene signature or MapQuant Dx. Fig 3 illustrates our thoughts on how characterisation of luminal type breast cancers can be further enhanced with new markers.

Within the last couple of decades there has been a significant improvement in the understanding of the ER pathway. This has revealed the complexity of the pathway, as well as identifying key molecules that play an important function in modulating its function. However, several areas of ER biology are still poorly understood; these include the function of the ER in the cytoplasm/plasma membrane, its role in the differentiation to proliferation switch, and pathways associated with resistance to hormonal therapy. Better understanding of these areas will permit better classification and a personalised approach to management of ER-positive breast cancers.

\section{Take-home messages}

- Oestrogen-receptor (ER)-positive tumours are heterogeneous, and the efficacy of hormonal therapy depends on status of multiple other proteins including other transcription factors such as FOXA1, GATA-3, growth factors, and co-activator and co-repressor proteins.

- Expression of ER by immunohistochemistry is strongly correlated with quantitative reverse transcriptase-PCR and immunohistochemistry remains the method of choice for hormone receptor analysis.

- A number of newer assays are being developed for prognostication of ER-positive tumours.

Acknowledgements: The laboratory of $\mathrm{H}$ Nakshatri is supported by grants from the National Institutes of Health (CA89153, CA123561), the Susan G Komen Foundation (BCTR0601111) and the IU Simon Cancer Center Breast Cancer Pilot Grant.

Competing interests: None declared.

\section{REFERENCES}

1. Anderson E. The role of oestrogen and progesterone receptors in human mammary development and tumorigenesis. Breast Cancer Res 2002;4:197-201.

2. Clarke RB, Anderson E, Howell A. Steroid receptors in human breast cancer. Trends Endocrinol Metab 2004;15:316-23.

3. Kuiper GG, Enmark E, Pelto-Huikko M, et al. Cloning of a novel receptor expressed in rat prostate and ovary. Proc Natl Acad Sci U S A 1996;93:5925-30.

4. Mosselman S, Polman J, Dijkema R. ER $\beta$ : identification and characterization of a novel human estrogen receptor. FEBS Lett 1996;392:49-53.

5. Tremblay GB, Tremblay A, Copeland NG, et al. Cloning, chromosomal localization, and functional analysis of the murine estrogen receptor beta. Mol Endocrinol 1997:11:353-65. 
6. Moore JT, McKee DD, Slentz-Kesler K, et al. Cloning and characterization of human estrogen receptor beta isoforms. Biochem Biophys Res Commun 1998;247:75-8.

7. Chen YC, Kraft P, Bretsky P, et al. Sequence variants of estrogen receptor beta and risk of prostate cancer in the National Cancer Institute Breast and Prostate Cancer Cohort Consortium. Cancer Epidemiol Biomarkers Prev 2007;16:1973-81.

8. Ali S, Coombes RC. Endocrine-responsive breast cancer and strategies for combating resistance. Nat Rev 2002;2:101-12.

9. Arpino G, Wiechmann L, Osborne CK, et al. Crosstalk between the estrogen receptor and the HER tyrosine kinase receptor family: molecular mechanism and clinical implications for endocrine therapy resistance. Endocr Rev 2008;29:217-33.

10. Shiau AK, Barstad D, Loria PM, et al. The structural basis of estrogen receptor/ coactivator recognition and the antagonism of this interaction by tamoxifen. Cell 1998;95:927-37.

11. Dauvois S, Danielian PS, White R, et al. Antiestrogen ICl 164,384 reduces cellular estrogen receptor content by increasing its turnover. Proc Natl Acad Sci U S A 1992:89:4037-41.

12. McKenna NJ, O'Malley BW. Combinatorial control of gene expression by nuclear receptors and coregulators. Cell 2002;108:465-74.

13. Lonard DM, Lanz RB, O'Malley BW. Nuclear receptor coregulators and human disease. Endocr Rev 2007;28:575-87.

14. Lonard DM, O'Malley BW. Nuclear receptor coregulators: judges, juries, and executioners of cellular regulation. Mol Cell 2007;27:691-700.

15. Osborne CK, Bardou V, Hopp TA, et al. Role of the estrogen receptor coactivator AIB1 (SRC-3) and HER-2/neu in tamoxifen resistance in breast cancer. J Natl Cancer Inst 2003;95:353-61.

16. Thorat M, Turbin D, Morimiya A, et al. Amplified in breast cancer 1 (AIB1) expression in breast cancer. Histopathology. In press.

17. Green AR, Burney C, Granger CJ, et al. The prognostic significance of steroid receptor co-regulators in breast cancer: co-repressor NCOR2/SMRT is an independent indicator of poor outcome. Breast Cancer Res Treat 2008;110:427-37.

18. Kushner PJ, Agard DA, Greene GL, et al. Estrogen receptor pathways to AP-1. J Steroid Biochem Mol Biol 2000;74:311-7.

19. Kushner PJ, Agard D, Feng WJ, et al. Oestrogen receptor function at classical and alternative response elements. Novartis Found Symp 2000;230:20-6. Discussion in Novartis Found Symp 2000;230:27-40

20. Frasor J, Danes JM, Komm B, et al. Profiling of estrogen up- and down-regulated gene expression in human breast cancer cells: insights into gene networks and pathways underlying estrogenic control of proliferation and cell phenotype. Endocrinology 2003;144:4562-74.

21. Levin ER, Pietras RJ. Estrogen receptors outside the nucleus in breast cancer. Breast Cancer Res Treat 2008;108:351-61.

22. Kim HP, Lee JY, Jeong JK, et al. Nongenomic stimulation of nitric oxide release by estrogen is mediated by estrogen receptor alpha localized in caveolae. Biochem Biophy Res Commun 1999;263:257-62.

23. Kelly MJ, Levin ER. Rapid actions of plasma membrane estrogen receptors. Trends Endocrinol Metab 2001;12:152-6.

24. Shou J, Massarweh S, Osborne CK, et al. Mechanisms of tamoxifen resistance: increased estrogen receptor-HER2/neu cross-talk in ER/HER2-positive breast cancer. J Natl Cancer Inst 2004;96:926-35.

25. Schafer JM, Bentrem DJ, Takei $\mathrm{H}$, et al. A mechanism of drug resistance to tamoxifen in breast cancer. J Steroid Biochem Mol Biol 2002;83:75-83.

26. Dowsett M, Houghton J, Iden C, et al. Benefit from adjuvant tamoxifen therapy in primary breast cancer patients according oestrogen receptor, progesterone receptor, EGF receptor and HER2 status. Ann Oncol 2006;17:818-26.

27. Dowsett M, Allred C, Knox J, et al. Relationship between quantitative estrogen and progesterone receptor expression and human epidermal growth factor receptor 2 (HER-2) status with recurrence in the Arimidex, Tamoxifen, Alone or in Combination trial. J Clin Oncol 2008;26:1059-65.

28. Tonetti DA, Morrow M, Kidwai N, et al. Elevated protein kinase $\mathrm{C}$ alpha expression may be predictive of tamoxifen treatment failure. Br J Cancer 2003;88:1400-2.

29. Carroll JS, Liu XS, Brodsky AS, et al. Chromosome-wide mapping of estrogen receptor binding reveals long-range regulation requiring the forkhead protein FoxA1. Cell 2005; 122:33-43.

30. Anderson MK. At the crossroads: diverse roles of early thymocyte transcriptional regulators. Immunol Rev 2006;209:191-211.

31. Cirillo LA, Lin FR, Cuesta I, et al. Opening of compacted chromatin by early developmental transcription factors HNF3 (FoxA) and GATA-4. Mol Cell 2002;9:279-89.

32. Jenssen TK, Kuo WP, Stokke T, et al. Associations between gene expressions in breast cancer and patient survival. Hum Genet 2002;111:411-20.

33. Hoch RV, Thompson DA, Baker RJ, et al. GATA-3 is expressed in association with estrogen receptor in breast cancer. Int J Cancer 1999;84:122-8.

34. Jacquemier $\mathbf{J}$, Ginestier $\mathbf{C}$, Rougemont $\mathbf{J}$, et al. Protein expression profiling identifies subclasses of breast cancer and predicts prognosis. Cancer Res 2005;65:767-79.

35. Mehra R, Varambally S, Ding L, et al. Identification of GATA3 as a breast cancer prognostic marker by global gene expression meta-analysis. Cancer Res 2005:65:11259-64

36. Kaestner KH. The hepatocyte nuclear factor 3 (HNF3 or FOXA) family in metabolism. Trends Endocrinol Metab 2000;11:281-5.

37. Lupien M, Eeckhoute J, Meyer CA, et al. FoxA1 translates epigenetic signatures into enhancer-driven lineage-specific transcription. Cell 2008;132:958-70.
38. Laganiere $\mathbf{J}$, Deblois $\mathrm{G}$, Lefebvre $\mathbf{C}$, et al. From the cover: location analysis of estrogen receptor alpha target promoters reveals that FOXA1 defines a domain of the estrogen response. Proc Natl Acad Sci U S A 2005;102:11651-6.

39. Badve S, Turbin D, Thorat MA, et al. FOXA1 expression in breast cancercorrelation with luminal subtype A and survival. Clin Cancer Res 2007;13:4415-21.

40. Thorat MA, Marchio C, Morimiya A, et al. Forkhead box A1 expression in breast cancer is associated with luminal subtype and good prognosis. J Clin Path 2008;61:327-32.

41. Wolf I, Bose S, Williamson EA, et al. FOXA1: Growth inhibitor and a favorable prognostic factor in human breast cancer. Int J Cancer 2007;120:1013-22.

42. Habashy H0, Powe DG, Rakha EA, et al. Forkhead-box A1 (FOXA1) expression in breast cancer and its prognostic significance. Eur J Cancer 2008;44:1541-51.

43. Shoker BS, Jarvis C, Clarke RB, et al. Estrogen receptor-positive proliferating cells in the normal and precancerous breast. Am J Pathol 1999;155:1811-5.

44. Shoker BS, Jarvis C, Clarke RB, et al. Abnormal regulation of the oestrogen receptor in benign breast lesions. J Clin Path 2000;53:778-83.

45. Ewan KB, Oketch-Rabah HA, Ravani SA, et al. Proliferation of estrogen receptoralpha-positive mammary epithelial cells is restrained by transforming growth factorbeta1 in adult mice. Am J Pathol 2005;167:409-17.

46. Hopp TA, Weiss HL, Hilsenbeck SG, et al. Breast cancer patients with progesterone receptor PR-A-rich tumors have poorer disease-free survival rates. Clin Cancer Res 2004; 10:2751-60.

47. Paik S, Shak S, Tang G, et al. Expression of the 21 genes in the Recurrence Score assay and tamoxifen clinical benefit in the NSABP study B-14 of node negative, estrogen receptor positive breast cancer. J Clin Oncol 2005;23(16S):6s.

48. Cui X, Schiff R, Arpino G, et al. Biology of progesterone receptor loss in breast cancer and its implications for endocrine therapy. J Clin Oncol 2005;23:7721-35.

49. MacFarlane R, Speers $\mathrm{CH}$, Masoudi H, et al. Molecular changes in the primary breast cancer versus the relapsed/metastatic lesion from a large population-based database and tissue microarray series [abstract]. J Clin Oncol 2008;26:41s.

50. Broglio K, Moulder S, Hsu L, et al. Prognostic impact of discordance/concrdance of triple-receptor expression between primary tumor and metastasis in patients with metastatic breast cancer [abstract]. J Clin Oncol 2008;26:41s.

51. Cheang MC, Treaba DO, Speers CH, et al. Immunohistochemical detection using the new rabbit monoclonal antibody SP1 of estrogen receptor in breast cancer is superior to mouse monoclonal antibody 1D5 in predicting survival. J Clin Oncol 2006;24:5637-44.

52. Badve SS, Baehner FL, Gray RP, et al. Estrogen- and progesterone-receptor status in ECOG 2197: comparison of immunohistochemistry by local and central laboratories and quantitative reverse transcription polymerase chain reaction by central laboratory. J Clin Oncol 2008;26:2473-81.

53. Baehner FL, Maddala T, Alexander C, et al. A Kaiser Permanente population-based study of ER and PR expression by standard method, immunohistochemistry (IHC), compared to a new method, quantitative reverse transcriptase polymerase chain reaction (RT-PCR) [abstract]. Mod Pathol 2007;21:20A.

54. Allred DC, Harvey JM, Berardo M, et al. Prognostic and predictive factors in breast cancer by immunohistochemical analysis. Mod Pathol 1998;11:155-68.

55. Harvey JM, Clark GM, Osborne CK, et al. Estrogen receptor status by immunohistochemistry is superior to the ligand-binding assay for predicting response to adjuvant endocrine therapy in breast cancer. J Clin Oncol 1999;17:1474-81.

56. Paik S, Shak S, Tang G, et al. A multigene assay to predict recurrence of tamoxifentreated, node-negative breast cancer. New Engl J Med 2004;351:2817-26.

57. Paik S, Tang G, Shak S, et al. Gene expression and benefit of chemotherapy in women with node-negative, estrogen receptor-positive breast cancer. J Clin Oncol 2006;24:3726-34

58. Perou CM, Sorlie T, Eisen MB, et al. Molecular portraits of human breast tumours Nature 2000:406:747-52.

59. Cheang $\mathbf{M}$, Voduc D, Tyldesley $\mathbf{S}$, et al. Breast cancer molecular subtypes and locoregional recurrence [abstract]. J Clin Oncol 2008;26:9s.

60. Parker J, Mullins $\mathrm{M}$, Cheang $\mathrm{M}$, et al. A supervised risk predictor of breast cancer based on biologic subtypes [abstract]. J Clin Oncol 2008;26:580s.

61. Albain K, Barlow W, Shak S, et al. Prognostic and predictive values of the 21-gene recurrence score assay in postmenopausal, node-positive, ER-positive breast cancer (S8814, INT0100) [abstract]. Proceedings of the 30th San Antonio Breast Cancer Symposium; 14-17 December 2007, San Antonio, Texas. San Antonio: Baylor College of Medicine and the American Association for Cancer Research, 2007.

62. Goetz MP, Suman VJ, Ingle JN, et al. A two-gene expression ratio of homeobox 13 and interleukin-17B receptor for prediction of recurrence and survival in women receiving adjuvant tamoxifen. Clin Cancer Res 2006;12:2080-7.

63. Ma XJ, Salunga R, Dahiya $S$, et al. A five-gene molecular grade index and HOXB13:IL17BR are complementary prognostic factors in early stage breast cancer Clin Cancer Res 2008;14:2601-8.

64. Ma XJ, Wang Z, Ryan PD, et al. A two-gene expression ratio predicts clinical outcome in breast cancer patients treated with tamoxifen. Cancer Cell 2004;5:607-16.

65. Ignatiadis $\mathbf{M}$, Sotiriou C. Understanding the molecular basis of histologic grade. Pathobiology 2008;75:104-11.

66. Loi S, Haibe-Kains B, Desmedt C, et al. Definition of clinically distinct molecular subtypes in estrogen receptor-positive breast carcinomas through genomic grade. J Clin Oncol 2007:25:1239-46.

67. Kouros-Mehr H, Slorach EM, Sternlicht MD, et al. GATA-3 maintains the differentiation of the luminal cell fate in the mammary gland. Cell 2006;127:1041-55

68. Kouros-Mehr H, Bechis SK, Slorach EM, et al. GATA-3 links tumor differentiation and dissemination in a luminal breast cancer model. Cancer cell 2008;13:141-52. 